\title{
Stab Wound Extension for the Eviscerated Small Bowel with Severe Ischemic Changes
}

\author{
Chan Kyu Lee, M.D.*, Chan Yong Park, M.D., Ph.D. ${ }^{\dagger}$, Kwang Hee Yeo, M.D.*, Ho Hyun Kim, M.D.* \\ *Department of Trauma Surgery, Pusan National University Hospital, Busan, \\ ${ }^{\dagger}$ Department of Trauma Surgery, Wonkwang University Hospital, Iksan, Korea
}

Correspondence to:
Kwang Hee Yeo, M.D.
Department of Trauma Surgery,
Pusan National University
Hospital, 179 Gudeok-ro,
Seo-gu, Busan 49241, Korea
Tel: +82-51-240-7369
Fax: +82-51-240-7719
E-mail: songyu0428@gmail.com
ORCID:
http://orcid.org/0000-0003-1392-9196
An abdominal stab wound with evisceration is an indication of an emergency laparotomy. We encountered a case of a very severe ischemic change in the eviscerated small bowel as a result of stabbing. The patient was considered to have a high possibility of progressing to strangulation of the small bowel. Therefore, a stab wound extension was performed as a decompression in the emergency department before definite surgery in the operating room. Most of the small bowel could be saved except for the segment with the damage caused by the stab injury. The patient was discharged without complications. (J Acute Care Surg 2018;8:71-73)

Key Words: Abdominal stab wound, Eviscerated small bowel, Decompressive wound extension, Emergency room

Received July 6, 2017, Revised June 20, 2018, Accepted September 22, 2018

Copyright $(\subset 2018$ by Korean Society of Acute Care Surgery

(c) This is an Open Access article distributed under the terms of the Creative Commons Attribution Non-Commercial License (http://creativecommons.org/licenses/by-nc/4.0) which permits unrestricted non-commercial use, distribution, and reproduction in any medium, provided the original work is properly cited.

\section{Introduction}

The bowel evisceration can cause ischemic damage due to impaired blood circulation by abdominal wall muscle contraction. If a large amount of the small bowel is eviscerated, it is to proceed to strangulation [1]. Small bowel evisceration after stab wound injury, definitive surgery at the operation room (OR) is usually preferred because of the problem related to further contamination and damage to the eviscerated bowel. In our case, although the size of the stab wound was only $5 \mathrm{~cm}$, there was an obvious ischemic change to the strangulated small bowel, because more than $50 \mathrm{~cm}$ of ileum had been eviscerated. We decided to immediately extend the opening of the wound from $5 \mathrm{~cm}$ to $20 \mathrm{~cm}$ at the emergency room to restore blood circulation and brought the patient to the OR for definite surgery.

\section{Case Report}

A 44-year-old man was presented to the emergency department with a bowel evisceration after stabbing in the left side of the abdomen. He took 33 minutes to the emergency room. His vital signs were as follows: blood pressure $90 / 60 \mathrm{mmHg}$, pulse rate 101 beats/min, respiration rate 20 breaths/min, body temperature $36.2^{\circ} \mathrm{C}$, oxygen saturation $96 \%$. A large amount of small bowel was eviscerated. And eviscerated bowel showed severe ischemic change and multiple transections (Fig. 1). The systolic blood pressure decreased to $60 \mathrm{mmHg}$ after admission, ischemic changed small bowel deteriorated, endotracheal tube insertion was performed 4 minutes after admission. Our emergency room have laparotomy and thoracotomy devices (operation set), so we improved the circulation by performing a stab wound extension as a decompression in the emergency department to prevent 
strangulation of the small bowel (Fig. 2). In addition, ligation of the bleeding arteries of the mesentery was performed. And then was rapping bowel by gauzes, abdominal skin wound by IOBAN $^{3 \mathrm{M}}$. Laboratory examination revealed WBC $9.19 \times 10^{3} / \mu \mathrm{l}$, Hb $13.6 \mathrm{~g} / \mathrm{dl}$, Platelet $226 \times 10^{3} / \mu \mathrm{l}$, Lactic acid $2.0 \mathrm{mmol} / \mathrm{L}$, Base Excess 3.0. The patient was transferred to the operating room. The circulation of the eviscerated small bowel was improved (Fig. 3). Multiple transections were observed in the jejunum and the ileum. Therefore segmental resection and anastomosis were performed (Fig. 4). Thus, most of the small bowel could be saved except for the segment with damage caused by stab injury.

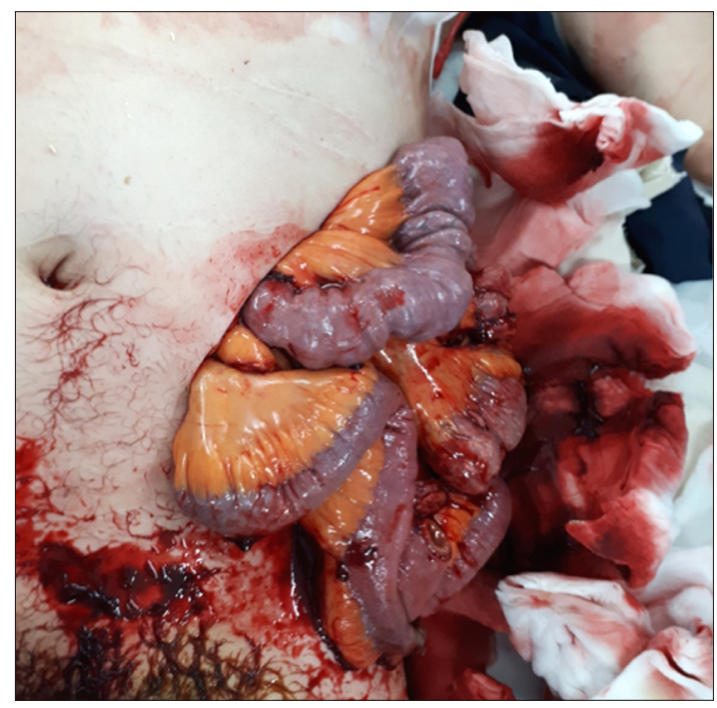

Fig. 1. A large amount of small bowel is eviscerated through the stab wound in the left side of the abdomen on arrival at the emergency department and ischemic change is very severe. Multiple transections of the small bowel are also observed.

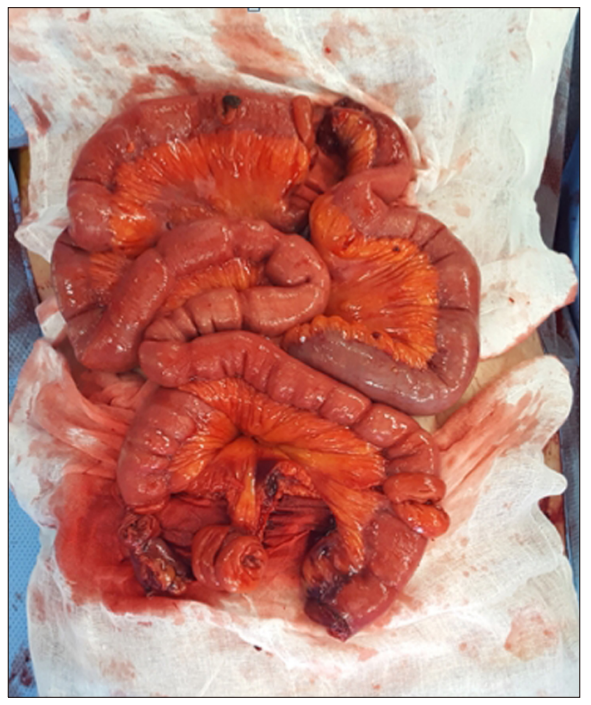

Fig. 3. Operative findings include multiple transection of the jejunum at the distal $100 \mathrm{~cm}$ from the Treitz ligament (upper) and the ileum at the proximal $150 \mathrm{~cm}$ from the ileocecal valve (lower).

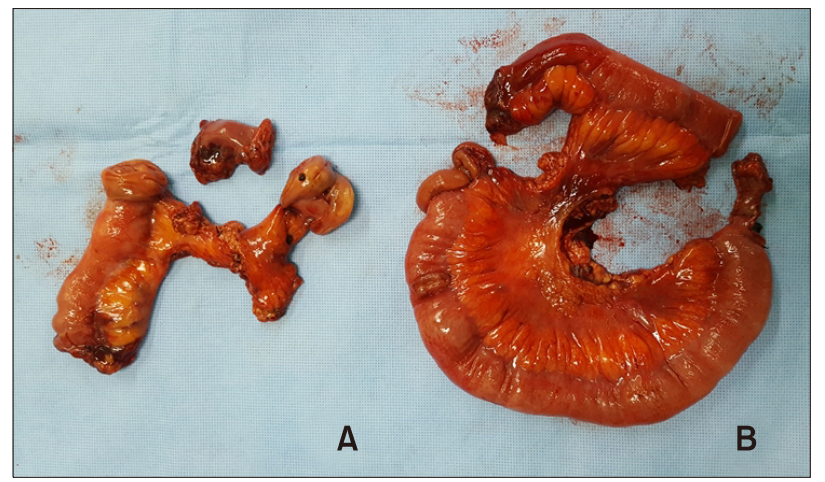

Fig. 4. After segmental resection of injured site of the jejunum (A) and the jejunum (B) is performed, improvement of ischemic change and multiple transections are observed.
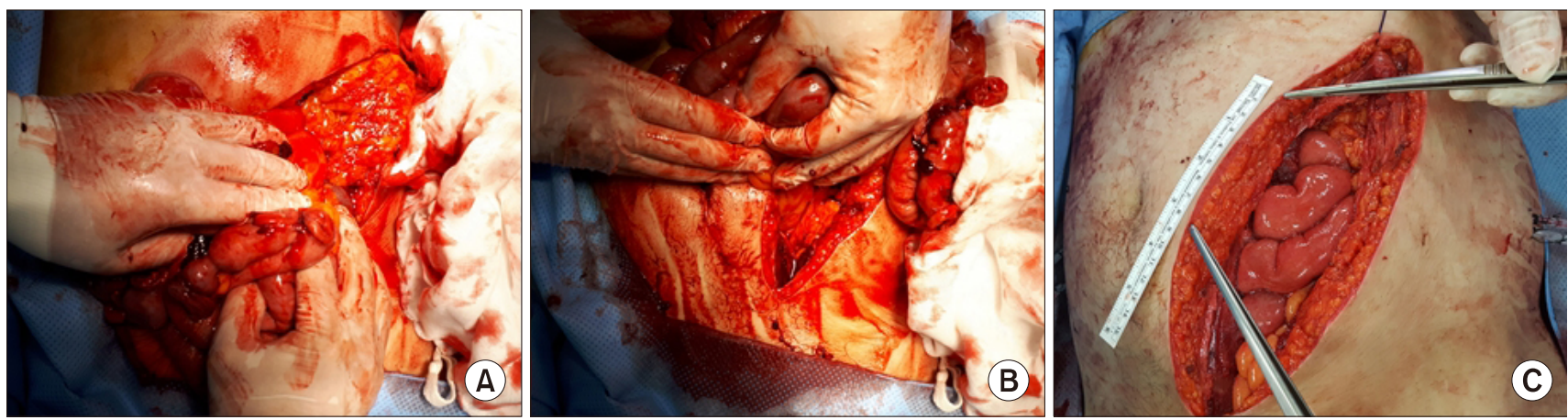

Fig. 2. (A, B) Stab wound extension for decompression is performed superiorly and inferiorly to prevent progressing to the strangulation of eviscerated small bowel with severe ischemic change in emergency department. (C) Initial stab wound is $8 \mathrm{~cm}$, wound extension is performed $8 \mathrm{~cm}$ superiorly and $10 \mathrm{~cm}$ inferiorly. 
The patient was discharged without any complications.

\section{Discussion}

Various organ eviscerations occur after abdominal stab injury. Evisceration is recognized as an indication of immediate laparotomy [2-4]. Bowel evisceration may cause ischemic change due to circulatory problem. If a large amount of the small bowel is eviscerated, it is likely to proceed to strangulation [1]. In this case, it is important to decompress the eviscerated bowel as quickly as possible which can be exacerbated by painful abdominal wall muscle contractions. If the ischemic change is severe as like present case, it may be necessary to try decompressive wound extension in the emergency department to prevent getting worse during the transfer to the operating room.

\section{Acknowledgments}

This work was supported by Clinical Research grant from Pusan National University Hospital in 2018.

\section{Conflicts of Interest}

No potential conflict of interest relevant to this article was reported.

\section{References}

1. Biffl WL, Kaups KL, Cothren CC, Brasel KJ, Dicker RA, Bullard MK, et al. Management of patients with anterior abdominal stab wounds: a Western Trauma Association multicenter trial. J Trauma 2009;66:1294-301.

2. Nicholson K, Inaba K, Skiada D, Okoye O, Lam L, Grabo $\mathrm{D}$, et al. Management of patients with evisceration after abdominal stab wounds. Am Surg 2014;80:984-8.

3. Sanei B, Mahmoudieh M, Talebzadeh H, Shahabi Shahmiri S, Aghaei Z. Do patients with penetrating abdominal stab wounds require laparotomy? Arch Trauma Res 2013;2:21-5.

4. Seror, Minvielle, Delonca, Xerri. Evisceration of half of the small intestine loops following stab wound 12 hours old; strangulation of the small intestine at the site of the wound; severe state of shock; emergency intervention under transfusion, novocaine anesthesia \& levophed perfusion; postoperative hibernation therapy; recovery. Afr Fr Chir 1957; 15:291-2 